Research Article

\title{
Potential drug-drug interactions among hospitalized cardiac patients
}

\author{
Ajay D. Shanbhag ${ }^{1 *}$, Hema N. G. ${ }^{1}$, K. S. Sadananda ${ }^{2}$
}

${ }^{1}$ Department of Pharmacology, Mysore Medical College and Research Institute, Irwin road, Mysuru, India

${ }^{2}$ Department of Cardiology, Sri Jayadeva Institute of Cardiovascular Sciences and Research, Mysuru, India

Received: 04 August 2016 Accepted: 03 September 2016

\section{*Correspondence to:}

Dr. Ajay D. Shanbhag, Email: ajayshanbhag81@ gmail.com

Copyright: (C) the author(s), publisher and licensee Medip Academy. This is an openaccess article distributed under the terms of the Creative Commons Attribution NonCommercial License, which permits unrestricted noncommercial use, distribution, and reproduction in any medium, provided the original work is properly cited.

\begin{abstract}
Background: Drug-drug interactions (DDIs) are a major cause for concern in patients with cardiovascular disorders due to multiple co-existing conditions and the wide class of drugs they receive. The objective of our study was to identify potential drug-drug interactions among hospitalized cardiac patients and to identify the risk factors associated with these interactions.

Methods: After obtaining approval from Institutional Ethical Committee, a prospective observational study was carried out among 367 hospitalized cardiac patients in Sri Jayadeva Institute of Cardiovascular Sciences and Research, Mysuru. Cardiac patients prescribed at least 2 drugs and having hospital stay of more than 24 hour duration were enrolled into the study. The prescriptions were analysed for potential DDIs using MEDSCAPE multidrug interaction checker tool. Descriptive statistics, Student ' $t$ ' test, ANOVA and Pearson correlation coefficient were used to analyse the results.

Results: The incidence of potential DDIs was $98 \%$ with 360 prescriptions having at least one potential DDI. A total of 38 potentially interacting drug pairs were identified among which majority were of significant grade while only 3 were serious. Majority of interactions were pharmacodynamic $(76.3 \%)$ in nature. Aspirin/clopidogrel $(71.1 \%)$ and pantoprazole/clopidogrel $(69.8 \%)$ were the most common interacting pairs. Drugs most commonly involved were aspirin, clopidogrel, heparin, pantoprazole and ramipril. Age, female gender, polypharmacy, prolonged hospital stay, stay in ICU and diabetes mellitus were the risk factors found associated with the potential DDIs.

Conclusions: Proper therapeutic planning, routine monitoring of cardiac inpatients and usage of online DDI database will avoid potentially hazardous consequences in cardiac in-patients.
\end{abstract}

Keywords: DDIs, MEDSCAPE, Cardiac in-patients

\section{INTRODUCTION}

Drug-drug interactions (DDIs) represent a special category of adverse drug reactions in which the effects of one drug influence the effects of the other, thus either limiting effectiveness or inducing toxicity. Overall, $1 \%$ of hospital admissions and $16 \%$ of admissions due to ADRs can be attributed to DDIs. A higher number of DDIs is also significantly associated with longer hospitalization and higher treatment costs. ${ }^{1,2}$

Approximately 37 - $60 \%$ of patients admitted to hospital may have one or more potentially interacting drug combinations at admission. In inpatients, the risk of having potentially interacting drug combinations can additionally increase because new drugs are often added to the existing drug therapy. DDIs are a concern for patients and providers, as polypharmacy is becoming more common in managing complex diseases or comorbidities and the consequences can range from untoward effects to drug-related morbidity and mortality. Healthcare professionals' ability to recognize potential DDIs is important in reducing their potential risks and adverse consequences. ${ }^{3}$

Studies have revealed that DDIs are a major clinical problem along with other adverse drug reactions especially in the hospitalized cardiac patients. Cardiovascular patients are more often reported with potential drug-drug interactions (pDDIs) as compared to patients with other diseases. The possible reason behind higher pDDI rate in cardiovascular diseases may include 
elder age, multiple drug regimen, and pharmacokinetic or pharmacodynamic nature of drugs used in cardiology. ${ }^{4}$

Hence, this study was conducted to evaluate the pattern of potential drug-drug interactions and to identify the associated risk factors among hospitalized cardiac patients in Sri Jayadeva Institute of cardiovascular sciences and research, Mysuru.

\section{METHODS}

\section{Setting and study design}

After obtaining Institutional Ethical Committee approval, this prospective observational study was carried out in Sri Jayadeva Institute of Cardiovascular Sciences and Research, Mysuru. The study was carried out for a period of 3 months between August to October 2015.

\section{Study population}

Cardiac patients aged 18 years or older admitted to the cardiology unit with a hospital stay of at least 24 hours and those prescribed two or more drugs were enrolled for the study. Patients referred to the cardiology unit for evaluation, patients visiting on outpatient basis and those who died during hospital stay were excluded from the study

\section{Tools used}

Patient case record was used for collecting demographic and medication profile of patients. Computerised DDI database system (MEDSCAPE database) was used to identify and analyse the pattern of potential DDIs. MEDSCAPE contains a separate tool for detecting DDIs known as the multidrug interaction checker tool. On entering the drugs one by one, the program lists the possible DDIs and categorizes DDIs according to their interaction effect, severity (contra-indicated, serious, significant, and minor), and management. ${ }^{5} \mathrm{~A}$ serious interaction emphasizes the need to use an alternative treatment, significant interaction emphasizes the need to monitor the patient closely and minor interactions do no warrant any change in drug therapy.

\section{Operational modality}

The medications taken by the patients during their hospital stay were analysed for possible drug interaction via the electronic database - MEDSCAPE database. The type and severity of the identified interacting pairs was documented as per the database. Also, the risk factors associated with the potential DDIs were studied.

\section{Statistical analysis}

Descriptive statistics, Student ' $t$ ' test, ANOVA and Pearson correlation coefficient were used to analyse the results. Descriptive statistics were used for summarizing the demographic parameters and potential DDIs. Pearson correlation coefficient was used to find the association between the risk factors and potential DDIs. The statistical analysis was done using Statistical Package for Social Sciences, version 20.0.

\section{RESULTS}

A total of 367 cardiac inpatients who fulfilled the inclusion and exclusion criteria were enrolled into the study. Among them, prescriptions of 360 patients were found to have at least one potential interacting drug combination. The overall incidence rate was found to be $98 \%$.

\section{Demographic profile}

Out of the 367 patients enrolled, majority of the patients were in the age group of 51 - 60 years. The mean age group was $55 \pm 10.24$ years. A higher number of males (239 [65\%]) were enrolled compared to females (128 [35\%]). The most common diagnosis was acute coronary syndrome (235 [64\%]) followed by hypertension (201 [54.7\%]) and congestive heart failure (16\%) (Figure 1). Dyslipidemia (209 [57\%]) was the most common comorbidity followed by diabetes mellitus (101 [27.5\%]). A total of 2417 drugs were prescribed and the average number of drugs per prescription was 8.4. The length of hospital stay was found to be $4.7 \pm 1.8$ days. Among the 367 patients, 235 had to be managed in the ICU for at least a day during their stay in the hospital.

Table 1: Interacting drug-pairs of serious grade with their frequencies and potential consequences.

\begin{tabular}{|lll|}
\hline Interacting drug pair & $\begin{array}{l}\text { Frequency } \\
\mathbf{n = 3 6 7}\end{array}$ & $\begin{array}{l}\text { Potential } \\
\text { consequence }\end{array}$ \\
\hline Ceftriaxone/heparin & 61 & Bleeding \\
\hline Pantoprazole/digoxin & 29 & $\begin{array}{l}\text { Digoxin } \\
\text { toxicity }\end{array}$ \\
\hline Furosemide/gentamicin & 6 & $\begin{array}{l}\text { Gentamicin } \\
\text { toxicity } \\
\text { altered serum } \\
\text { potassium } \\
\text { levels }\end{array}$ \\
\hline $\mathbf{n}=$ sample size & & \\
\hline
\end{tabular}

Potential drug-drug interactions a total of 38 pDDIs were identified in this study. The total number of pDDIs per prescription was $6 \pm 3.1$. Majority of the identified pDDIs were due to pharmacodynamic mechanisms (29 [76.3\%]) while the remaining were caused either by pharmacokinetic interactions $(6[15.8 \%])$ or by a combination of pharmacokinetic and dynamic interactions (3 [7.9\%]) (Figure 2). Based on the severity, while majority of the pDDIs were of significant grade $(30$ [78.9\%]), $3(7.9 \%)$ were identified to be serious, $2(5.3 \%)$ were minor and the remaining were classified as both significant and minor (3 [7.9\%]). The interacting pairs of serious grade along with the potentially hazardous effects are enlisted in Table 1. The most common interacting drug pairs were aspirin/clopidogrel (256 [71.1\%]) followed by pantoprazole/clopidogrel (251 [69.8\%]) and aspirin/heparin (220 [59.9\%]). 
Table 2: Frequency, severity and potential consequences of commonly observed interacting drug-pairs.

\begin{tabular}{|llll|}
\hline Interacting drug pair & Frequency $(\mathbf{n}=\mathbf{3 6 7})$ & $\begin{array}{l}\text { Severity } \\
\text { Significant }\end{array}$ & Botential consequence \\
\hline Aspirin/clopidogrel & 261 & Significant & Decreased clopidogrel effect \\
\hline Pantoprazole/clopidogrel & 256 & Significant & Bleeding \\
\hline Aspirin/heparin & 220 & Significant & Bleeding \\
\hline Heparin/clopidogrel & 216 & Significant & $\begin{array}{l}\text { Decreased metoprolol effect } \\
\text { hyperkalemia }\end{array}$ \\
\hline Aspirin/metoprolol & 155 & Significant & $\begin{array}{l}\text { Decreased ramipril effect } \\
\text { renal dysfunction }\end{array}$ \\
\hline Aspirin/ramipril & 147 & Significant & hyperkalemia \\
\hline Heparin/ramipril & 125 & Significant & $\begin{array}{l}\text { Furosemide toxicity } \\
\text { nephrotoxicity }\end{array}$ \\
\hline Ceftrixone/furosemide & 61 & Serious & Bleeding \\
\hline Ceftriaxone/heparin & 61 & Significant & Serum potassium abnormality \\
\hline Spironolactone/furosemide & 59 & for & .
\end{tabular}

$\mathrm{n}=$ sample size, $*$ monitor closely, $* *$ use an alternative. Significant $=$ need for close clinical and laboratory monitoring.

Serious $=$ need to use an alternative.

Table 3: Statistical analysis of patient characteristics with pDDIs.

\begin{tabular}{|c|c|c|c|c|}
\hline Patient charcteristics & Groups & Frequency $(\mathbf{N}=367)$ & Number of pDDIs (mean) & P value \\
\hline \multirow{4}{*}{ Age (years) } & $41-50$ & 112 & 5.6250 & \multirow{4}{*}{$<0.05^{*}$} \\
\hline & $51-60$ & 136 & 5.7868 & \\
\hline & $61-70$ & 93 & 6.4839 & \\
\hline & $<70$ & 26 & $7.1538^{*}$ & \\
\hline \multirow{2}{*}{ Sex } & Male & 239 & 5.7699 & \multirow{2}{*}{$<0.05^{* *}$} \\
\hline & Female & 128 & 6.4609 & \\
\hline \multirow{2}{*}{ ICU stay (>/- 24 hours ) } & Yes & 235 & 6.9277 & \multirow{2}{*}{$<0.001^{* *}$} \\
\hline & No & 132 & 4.3788 & \\
\hline \multirow{3}{*}{ Duration of hospital stay } & $<3$ days & 8 & 1.8750 & \multirow{3}{*}{$<0.001^{*}$} \\
\hline & 3-5 days & 230 & 5.1348 & \\
\hline & $>5$ dys & 129 & 7.8295 & \\
\hline \multirow{3}{*}{ Number of drugs prescribed } & Up to 5 & 31 & 2.4194 & \multirow{3}{*}{$<0.001 *$} \\
\hline & $6-10$ & 287 & 5.9094 & \\
\hline & $11-20$ & 49 & 8.8776 & \\
\hline \multirow{2}{*}{ Diabetes } & Yes & 101 & 7.3960 & \multirow{2}{*}{$\begin{array}{l}< \\
0.001 * *\end{array}$} \\
\hline & No & 266 & 5.4850 & \\
\hline
\end{tabular}

* P value obtained after using ANOVA to compare the difference in means between groups; ** $\mathrm{P}$ value obtained after using Student ${ }^{\prime} t$ ' test to compare the difference in means between groups.

Table 4: Risk factor correlation with pDDIs.

\begin{tabular}{|lll|}
\hline Risk factor & Pearson correlation $(\mathbf{r})$ value & P value $^{*}$ \\
\hline Age & 0.136 & $<0.01$ \\
\hline Length of hospital stay & 0.506 & $<0.01$ \\
\hline Number of drugs per prescription & 0.620 & $<0.01$
\end{tabular}

*P value obtained by Pearson Correlation coefficient and $\mathrm{P}<0.01$ considered statistically significant. 


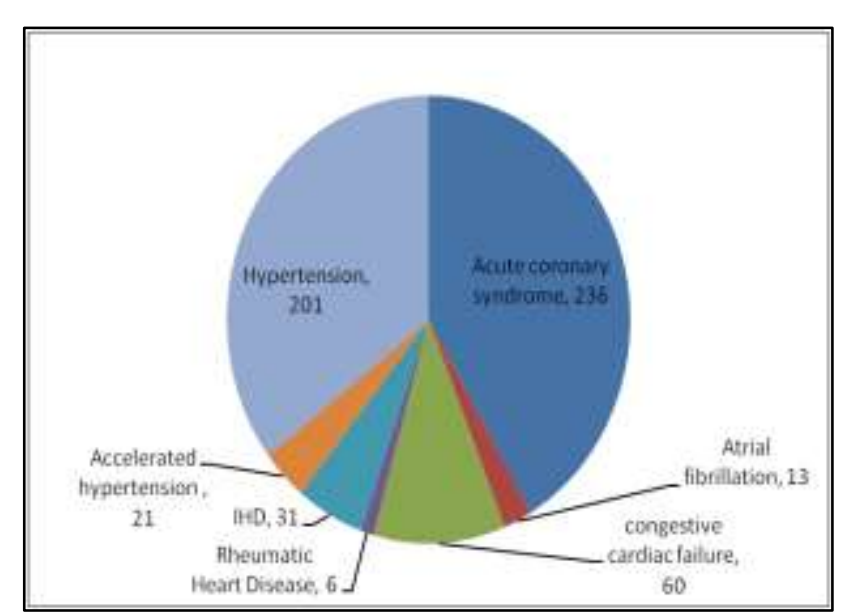

Figure 1: Diagnosis of cardiac in-patients. $n=367$ ( $n$ = sample size).

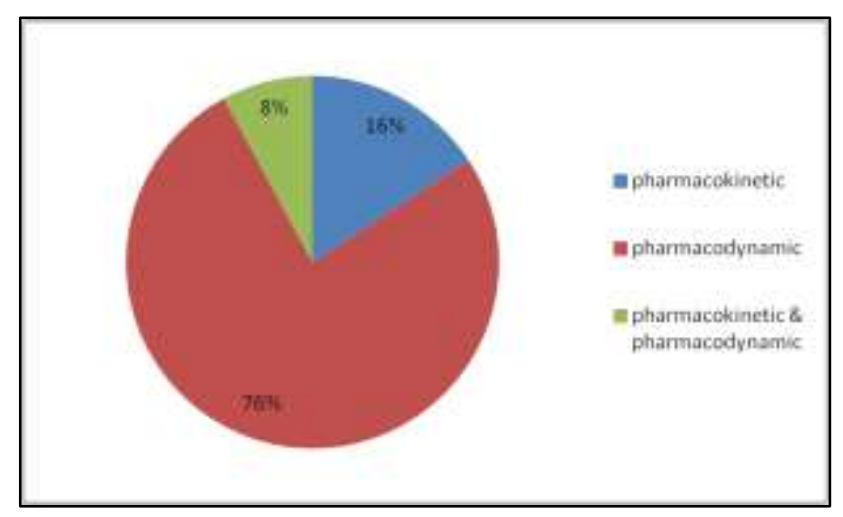

Figure 2: Type of DDIs.

Table 2 shows the list of some commonly observed interacting drug pairs along with their potential consequences. The pDDI involving aspirin (12 [31.6\%]) was the highest among all followed by furosemide (10 $[26.3 \%])$, spironolactone $(7[18.4 \%])$ and heparin $(5$ [13.2\%]).

\section{Risk factors}

Student ' $t$ ' test, ANOVA and Pearson correlation coefficient showed that age, female gender, duration of hospital stay, stay in ICU for a minimum duration of 24 hours, number of medicines prescribed and presence of diabetes mellitus had a significant influence on the incidence of pDDIs in our study. Table 3 and Table 4 summarize the statistical analysis of the risk factors associated with pDDIs in this study.

\section{DISCUSSION}

Drug-drug interactions (DDIs) are defined as two or more drugs interacting in such a manner that the effectiveness or toxicity of one or more drugs is altered on administration of the other. ${ }^{6}$ It can occur either pharmacokinetically or pharmacodynamically. Pharmacokinetic interaction occurs when either of the concurrently administered drugs have potential to alter other's pattern of absorption, distribution, metabolism and excretion. Similarly, pharmacodynamic interaction occurs if concurrently administered drugs have similar or opposite effects. $^{7}$

Cardiovascular diseases have been gaining importance in India recently because of increased incidence of the disease over the years. It is the first among top five causes of deaths in Indian population. ${ }^{8,9}$ The country wise statistics of the WHO on non-communicable diseases (NCDs) estimates that NCDs account for $53 \%$ of the total deaths in India, out of which CVDs have a major share of $24 \%{ }^{9}$

Drug-drug interactions may be beneficial or harmful. Harmful drug-drug interactions are important as they cause $10-20 \%$ of the adverse drug reactions requiring hospitalisation and they can be avoided. ${ }^{10}$ The potential benefits of drug combinations should be weighed against the seriousness of the DDI, taking into account the availability of alternatives. If the benefit of treatment is of such importance that it outweighs the potential risks, and no safer alternatives are apparent, then the risks of a potential DDI may be tolerated and treatment continued. ${ }^{3}$

The present study identified the pattern of pDDIs among patients admitted to Sri Jayadeva Institute of Cardiovascular sciences, Mysuru. The incidence rate of pDDIs was $98 \%$. Studies done by Patel et al. ${ }^{11}$ in a south Indian hospital and by Sharma et al. ${ }^{7}$ in the cardiac ward of a hospital in Nepal have shown prevalence rates $30.67 \%$ and $21.3 \%$ respectively. A similar study carried out in department of cardiology of the Ayub teaching hospital (ATH), Abbottabad showed a prevalence rate of $91.6 \%$. $^{4}$ The reason for a higher incidence of pDDIs in our study could be due to consideration of all grades of pDDIs, inclusion of patients in the Intensive Care Unit and the variation in the prescribing pattern of cardiologists in various hospitals.

The present study showed a higher incidence of pDDIs in females compared to males which were statistically significant. This finding is consistent with the results of a study done by Mateti et al. ${ }^{6}$ but differs from a study done by Murtaza et al. ${ }^{4}$ which showed no significant association between pDDIs and gender. Also, the incidence rate of pDDIs showed an increasing trend with age and this could be because the mean number of drugs per prescription was higher in the elderly patients due to coexisting co-morbidities. A study done by Kashyap et al shows similar findings. ${ }^{12}$

In the present study, a higher number of observed pDDIs were due to pharamacodynamic mechanisms (76.3\%) compared to pharmacokinetic type of interactions (15.8\%). These findings differ from those reported by Vonbach et al, Aparasu et al and Sharma et al. ${ }^{7,13,14}$ This 
difference may be due to differing patterns of prescription in various settings where the studies were conducted.

On analysing the severity of the interactions, majority of the identified pDDIs were of significant grade $(78.9 \%)$ as per the MEDSCAPE database. Only three interactions were found to be of severe grade $(7.9 \%)$. These results correlate well with the observations made in other studies where different electronic software was used to identify the pDDIs and majority of the identified interactions were of moderate severity., ${ }^{4,7}$

The most common interacting drug pairs were aspirin/clopidogrel (256 [71.1\%]) followed by pantoprazole/clopidogrel (251 [69.7\%]), aspirin/heparin (220 [59.9\%]), heparin/clopidogrel (216 [58.9\%]), aspirin/metoprolol (155 [42.2\%]), aspirin/ramipril (147 [40.1\%]) and heparin/ramipril (125 [34.1\%]). The pDDI involving aspirin (12 [31.6\%]) was the highest among all followed by furosemide (10 [26.3\%]), spironolactone (7 [18.4\%]) and heparin (5 [13.2\%]). These findings are similar to study done by Patel et al.11 and Smithburger et al. ${ }^{15}$ but differ from observations done by Sharma et al. ${ }^{7}$ where atorvastatin and enalapril were the most common drugs involved in DDIs.

Patients who were admitted to the ICU had a significantly higher incidence of pDDIs than the patients who were managed in the wards. This observation regarding the influence of ICU management on the incidence of drugdrug interactions correlates well with the observation made by Rodrigues AT et al. A highly complex environment of the ICU combined with the need for prescribing multiple drugs can predispose to increased likelihood of pDDIs in the prescriptions of patients managed in the ICU. ${ }^{16}$

The length of hospital stay in our study was $4.7 \pm 1.8$ days. A significant positive linear relationship was found between the length of hospital stay and pDDIs which correlates well with a study done by Moura et al. ${ }^{17}$ The reason for such an observation can be attributed to increasing drugs per prescription with increased number of days in the hospital.

Similar positive linear relationship was also found between the number of medicines prescribed and pDDIs and also between diabetes mellitus and pDDIs. As shown by many previous studies, these findings show that polypharmacy is clearly an independent risk factor for the development of drug-drug interactions. ${ }^{1,7,18}$

Since our study was only a prescription analysis, we couldn't comment whether the observed pDDIs actually led to clinically appreciably consequences or not. In a study done by Taegtmeyer et al. ${ }^{19}$ the authors have shown that pharmacologist agreed with only $11 \%$ of DDI alerts as shown by electronic software and $89 \%$ were not thought to be clinically significant. Hence, the major limitation in our study is the lack of clinical or laboratory evidence of the actual incidence of DDIs.

\section{CONCLUSION}

The high incidence of potential DDIs among cardiac inpatients in our study highlights the need to take appropriate measures to keep a check on some of the potentially hazardous consequences. Some of the potential consequences of the observed pDDIs were hemorrhage, alteration in serum potassium levels, hypoglycaemia, digoxin toxicity, nephrotoxicity and reduced efficacy of certain anti-hypertensive agents. Age, female gender, duration of hospital stay, stay in ICU for a minimum duration of 24 hours, number of medicines prescribed and presence of diabetes mellitus were the risk factors identified in this study.

One of the ways to minimize the consequences of DDIs would be to use the DDI database freely available online, both by clinicians as well as pharmacists. Also, proper therapeutic planning as well as routine monitoring of serum electrolytes, blood glucose and coagulation profile in cardiac in-patients is of utmost importance. At the same time confirmation of pDDIs clinically as well as by pharmacokinetic studies especially for significant and severe DDIs seems essential.

\section{ACKNOWLEDGEMENTS}

Authors would like to thank Dr. B. M. Parashivamurthy, Professor and HOD, Department of Pharmacology, Mysore Medical College and Research Institute for his guidance and support in carrying out this study. We would also extend our thanks to the entire staff of Sri Jayadeva Institute of cardiovascular sciences, Mysuru for their cooperation throughout the conduct of this study. Special thanks to our statistician, Dr. Lancy for helping us with the statistical analysis.

Funding: No funding sources Conflict of interest: None declared

Ethical approval: The study was approved by the Institutional Ethics Committee

\section{REFERENCES}

1. Cruciol-Souza JM, Thomson JC. Prevalence of potential drug-drug interactions and its associated factors in a Brazilian teaching hospital. J Pharm Pharmaceut Sci. 2006;9(3):427-33.

2. Roblek T, Trobec K, Mrhar A, Lainscak M. Potential drug-drug interactions in hospitalized patients with chronic heart failure and chronic obstructive pulmonary disease. Arch Med Sci. 2014;10(5):92032.

3. Kulkarni V, Bora SS, Sirisha S, Saji M, Sundaran S. A study on drug-drug interactions through prescription analysis in a South Indian teaching hospital. Ther Adv Drug Saf. 2013;4(4):141-6. 
4. Murtaza G, Khan MYG, Azhar S, Khan SA, Khan TM. Assessment of potential drug-drug interactions and its associated factors in the hospitalized cardiac patients. Saudi Pharm J. 2016;24:220-5.

5. Sivva D, Mateti UV, Neerati VM, Thiruthopu NS, Martha S. Assessment of drug-drug interactions in hypertensive patients at a superspeciality hospital. Avicenna J Med. 2015;5:29-35.

6. Mateti UV, Rajakannan T, Nekkanti H, Rajesh V, Mallaysamy SR, Ramachandran P. Drug-drug interactions in hospitalized cardiac patients. J Young Pharmacists. 2011;3:329-33.

7. Sharma S, Chhetri HP, Alam K. A study of potential drug-drug interactions among hospitalized cardiac patients in a teaching hospital in Western Nepal. Indian J Pharmacol. 2014;46:152-6.

8. Chauhan S, Aeri BT. Prevalence of cardiovascular disease in India and its economic impact - a review. International Journal of Scientific and Research Publications. 2013;10(3):1-5.

9. Chauhan S, Aeri BT. The rising incidence of cardiovascular diseases in India: assessing its economic impact. J Preventive Cardiology. 2015;4(4):735-40.

10. Snyder BD, Polasek TM, Doogue MP. Drug interactions: principles and practice. Aust Prescr. 2012;35(3):85-8.

11. Patel VK, Acharya LD, Rajakannan T, Surulivelrajan M, Guddattu V, Padmakumar R. Potential drug interactions in patients admitted to cardiology wards of a south Indian teaching hospital. Australas Med J. 2011;4:9-14.

12. Kashyap M, D’Cruz S, Sachdev A, Tiwari P. Drugdrug interactions and their predictors: results from
Indian elderly inpatients. Pharmacy Practice. 2013;11(4):191-5.

13. Vonbach P, Dubied A, Krähenbühl S, Beer JH. Prevalence of drug drug interactions at hospital entry and during hospital stay of patients in internal medicine. Eur J Intern Med. 2008;19:413-20.

14. Aparasu R, Baer R, Aparasu A. Clinically important potential drug-drug interactions in outpatient settings. Res Social Adm Pharm. 2007;3:426-37.

15. Smithburger PL, Kane Gill SL, Seybert AL. Drugdrug interactions in cardiac and cardiothoracic intensive care units: an analysis of patients in an academic medical centre in the US. Drug Saf. 2010;33:879-88.

16. Rodrigues AT, Stahlschmidt R, Granja S, Falcao ALE, Moriel P, Mazzola PG. Clinical relevancy and risks of potential drug-drug interactions in intensive therapy. Saudi Pharm J. 2015;23:366-70.

17. Moura C, Acurcio F, Belo N. Drug-drug interactions associated with length of stay and cost of hospitalization. J Pharm Pharmaceut Sci. 2009;12(3):266-72.

18. Dubova SV, Reyes Morales H, Torres Arreola Ldel P, Suárez Ortega M. Potential drug-drug and drug disease interactions in prescriptions for ambulatory patients over 50 years of age in family medicine clinics in Mexico City. BMC Health Serv Res. 2007;7:147.

19. Taegtmeyer A, Kullak-Ublick G, Widmer N, Falk V, Jetter, A. Clinical usefulness of electronic drug-drug interaction checking in the care of cardiovascular surgery inpatients. Cardiology. 2012;123:219-22.

Cite this article as: Shanbhag $\mathrm{AD}$, Hema NG, Sadananda KS. Potential drug-drug interactions among hospitalized cardiac patients. Int J Basic Clin Pharmacol 2016;5:2251-6. 